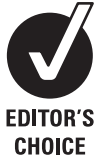

Correspondence to:

Bjørn Hofmann, Department of Health, Care and Nursing, University College of Gjøvik, PO Box 1, N-2802 Gjøvik, Norway bjoern.hofmann@hig.no and University of Oslo, Section for Medical Ethics, PO Box 1130, Blindern, N-0318 Oslo, Norway: b.m.hofmann@medisin.uio.no

Received 8 May 2009 Accepted 17 June 2009

\title{
Fallacies in the arguments for new technology: the case of proton therapy
}

\author{
B Hofmann
}

\section{ABSTRACT}

In a seminal article in the Journal of Medical Ethics, Søren Holm and Tuja Takala analysed two protechnology arguments in bioethics: the hopeful principle and the automatic escalator. They showed how these arguments relate to problematic arguments such as the precautionary principle and the empirical slippery slope argument, and argued that they should be used with great caution. The present article investigates the recent debate on proton beam therapy, where the hopeful principle and the automatic escalator are identified. However, the debate reveals a series of other arguments that deserve similar caution. An analysis of these arguments indicates that the roots of their fallacies are to be found in the ignorance of the uncertainties about risks and benefits and an overly optimistic attitude towards technology and progress. The point is not to argue against proton therapy, but rather to point out that flawed arguments for new technologies, such as proton therapy, can actually hamper their implementation instead of promoting it. Patients deserve the best technology available, not only on the basis of the best available evidence, but also on the basis of the best arguments.

In a seminal article, Søren Holm and Tuja Takala analysed two protechnology arguments in bioethics, namely, the hopeful principle and the automatic escalator. ${ }^{1}$ They showed how these arguments relate to problematic arguments such as the precautionary principle and the empirical slippery slope argument, and how the problems of the latter are relevant for the former. These four arguments (the hopeful principle, the automatic escalator, the precautionary principle and the empirical slippery slope argument) are all prevalent in ethical debates on new technologies with uncertain consequences, and Holm and Takala argued that they should be used with great caution. They pointed out that "Any analysis that argues from consequences must aim at taking on board all the risks of harm and all the possible benefits to all concerned, but it needs to do this in a way that properly acknowledges the uncertainties in predicting the effects of pursuing any technology."1

This article explores the recent debate on proton therapy, where both the automatic escalator and the hopeful principle are identified. In addition, it singles out a series of other protechnology arguments that on scrutiny turn out to be flawed. They merit the same caution as the arguments mentioned by Holm and Takala. Furthermore, the article investigates what these arguments have in common and why they seem so prevalent. In particular, it reveals how the arguments are based on great enthusiasm that tends to downplay uncertainties about risks and benefits and an overly optimistic attitude towards technology and progress.

Proton therapy is selected as an example due to the recent debate, ${ }^{2-12}$ but it is not a special case. We see similar arguments with many new technologies, especially costly high-tech ones. There have been fierce debates over MRI scanners during the 1980s and PET scanners during the 1990s, to mention only two. Such debates on emerging technologies tend to have a common structure, applying arguments that are logically or semantically unsound.

Hence, besides illustrating the relevance of the hopeful principle and the automatic escalator discussed by Holm and Takala, the article demonstrates how their call for cautiousness is relevant for other protechnology arguments as well, and how the flaws in the arguments are rooted in unwarranted enthusiasm and optimism. To identify and to be cautious with such arguments is important in order to provide good and just healthcare.

\section{PROTON THERAPY_ESCALATED HOPES}

Proton therapy is an external-particle-beam therapy in which protons are aimed at tumours. Because of the relatively high mass of protons, they do not scatter much and stay focused towards the target. This enables lower doses to affect the surrounding healthy tissue and provides a potential for reducing side effects and obtaining higher doses in the tumours. ${ }^{2}$

In the recent and interesting debate on the effectiveness of proton beam therapy, those in one branch of the debate have promoted the promulgation and reimbursement of proton therapy, ${ }^{6}$ whereas others have been more sceptical. ${ }^{5}$ Despite agreement that there are almost no randomised clinical trials that compare proton beam therapy and conventional $x$-ray therapy, different opinions about whether such trials are necessary, possible and ethical have sparked a lively debate. ${ }^{7-9}$ Among the discussed issues is whether the central requirement of equipoise can be satisfied in studies of proton therapy. ${ }^{7}$ One alleged reason why it is unnecessary to document the effectiveness (and efficiency) of proton therapy is that the "new technology" is not a new technology-only a modification of an existing technology (ie, radiotherapy), and thus does not have to be tested. ${ }^{12}$ Another one is that it is unnecessary, unacceptable and unethical to conduct randomised controlled trials (RCTs). ${ }^{6}$ 
The main argument of the debate is that targeted destruction of cancer cells is a good thing. Proton (beam) therapy has the advantage over ordinary radiotherapy that the beam can be controlled more precisely, so that less damage is done to healthy tissue. The effect of this has not yet been scientifically proven (by randomised trials). Nevertheless, proton therapy is so promising that we should implement it without further research or evidence.

At the core of this argument we can identify the hopeful principle: ${ }^{1}$

P1 (Premise 1) We know that B (eg, a cure for cancer) is a good thing.

P2 We suspect that y (eg, proton therapy) will lead to B, although this is not at present scientifically proven.

C (Conclusion) We conclude that, in the name of the hopeful principle, we should promote $y$ (even though it may turn out that it does not lead to B).

The argument resembles the precautionary principle, but with the opposite sign, and can in theory be used to promote any technology.

Furthermore, the argument against the need of RCTs to demonstrate the effectiveness of proton therapy can be structured as follows:

P1 There are epistemic and moral costs associated with promoting and pursuing a new technology $\mathrm{T}$ (eg, not having evidence from RCTs).

P2 Promoting the new technology T will, however, as a matter of empirical fact, lead to the good $U$ (improving cancer treatment).

P3 U (improving cancer treatment) is a great good.

C Therefore, we should pursue and promote $\mathrm{T}$, despite the epistemic and moral costs.

This can be recognised as the automatic escalator. It resembles the slippery slope argument, but with the opposite sign, and shares its weaknesses.

In addition to the hopeful principle and the automatic escalator, other arguments play a significant role in the debate on proton therapy.

\section{UNNECESSARY TO DOCUMENT THE EFFICIENCY OF NEW TECHNOLOGY}

One of these arguments is that "practitioners of proton beam therapy have found it ethically unacceptable to conduct RCTs comparing protons with x-rays". 6 The latter argument can be recognised in the literature on a wide range of technologies, ${ }^{13}$ and its structure is as follows:

P1 "The dose distributions that can be achieved with protons are in almost all cases superior to those possible with $x$ rays." (Protons deposit a third to half the energy in the uninvolved normal tissues outside the target volume compared with $x$ rays.) P2 "There is virtually no difference in tissue response per unit dose between protons of therapeutic energies as compared with $x$ rays, so that the only relevant differences are physical."

P3 "Radiation delivered to normal tissues causes damage to them ..."

P4 P1-3 are documented and generally accepted.

C1 There is a very "high probability that protons can provide superior therapy to that possible with $x$ rays in almost all circumstances".

P5 A comparison between proton and $x$-ray therapy "would not meet a central requirement for performing RCTs, namely that there be equipoise between the arms of the trial".

C2 Therefore it is unethical to perform RCTs to document the outcome of proton therapy. ${ }^{6}$
This has the form of petitio principii (also called circular argument, begging the question, assuming the answer, circulus in probando, and tautology). It assumes in the premise what one wants to prove in the conclusion, namely, that protons can provide better therapy than $x$ rays. The simplified form of the above argument is as follows:

P Proton therapy is superior to $x$-ray therapy.

C Therefore it is unnecessary (and unethical) to document the outcome of proton therapy compared with $x$-ray therapy.

It could also be argued that it is fallacious to draw the conclusion that it is unethical to document the outcome of proton therapy from the premise that protons are better than photons, because it is unclear what is meant by "better outcome". As there is a significant difference between efficacy, effectiveness and efficiency, this argument connects to the fallacy of equivocation if "better outcome" in the premise has a different meaning than in the conclusion. The form of the argument is as follows:

$\mathbf{P 1}$ It is unethical to perform RCTs comparing proton and $x$-ray therapy if it is known that proton therapy is better than $x$-ray therapy.

P2 Proton therapy is better than $x$-ray therapy.

C It is unethical to document the outcome of proton therapy. P1 holds if there already are RCTs that show the superiority of proton therapy. However, "better than" in P2 may refer to assumptions about mechanisms in physics and biology. A similar fallacy of equivocation may occur if P1 refers to documented efficacy, while P2 refers to effectiveness or even efficiency. Debates about what level of evidence is sufficient for implementing technologies are of course highly legitimate, but they should be as sound as possible. The point here is that if the meaning of "better" is different in P1 and P2, the argument is flawed.

\section{PROGRESS AND ANTICIPATED DECISION REGRET}

Another argument refers to the history of technology development and appeals to our belief in continued progress. The sceptical questions that historically were asked about technology that today is commonplace in every hospital seem ridiculous in hindsight. Correspondingly, our present scepticism will appear to be poor judgment in the future: "In our professional lives, we have lived to see almost identical arguments being made regarding new technologies, including the introduction of cobalt-60 teletherapy machines, the use of treatment simulators, the use of high-energy linear accelerators, the use of computed tomography, and so forth. We look back now on those arguments and wonder at the poor judgment that was evidenced then, and feel sure that history will judge the current controversy in the same manner."

This argument has the form:

P1 History shows that although we are sceptical to new technologies in the beginning, they are implemented and widely used in the end.

P2 Proton therapy is such a technology that meets scepticism, but that will be implemented and used in the end.

C We should implement proton therapy (now).

This argument is flawed because the first premise does not hold. There are many new technologies that have been met with scepticism (as well as technologies that were met with great optimism) that have not been implemented in the end: impedance tomography is but one example.

However, another interpretation of the argument has the following form: 
P1 If we do not implement $\mathrm{T}$ (eg, proton therapy), we will regret it.

P2 If we implement $\mathrm{T}$, we can avoid regretting the decision not to implement it.

C We should implement $\mathrm{T}$ (now).

This is an argument from anticipated decision regret. We decide now on the basis of what we expect will make us avoid regretting the decision in the future. The challenge here is of course whether P1 and P2 hold.

At the basis of these arguments there seems to be an appeal to novelty (or argumentum ad novitatem). It is assumed that the "new" technology is better than the predominant technology and that it will eventually be implemented and used:

P1 If $\mathrm{T}$ is a new technology, then $\mathrm{T}$ is better than the existing technology.

P2 $\mathrm{T}$ is a new technology.

C $\mathrm{T}$ should be implemented and used.

\section{BEING PROGRESSIVE}

Correspondingly, a frequent argument is that other countries that are comparable with our country have the technology in question, so we should implement it as well. Another version of the argument is that another country (or institution) that we compete with, or like to be ahead of, is on the verge of implementing a new technology, so we should do so quickly.

A negative version of such arguments is as follows: only a few other countries that are not comparable with our country do not have the technology. So we have to implement it. Luckily, these arguments seldom appear in specialised scientific journals, but they are used by the very same scientists who publish in these journals when they enter public debates. ${ }^{3}{ }^{12}$ One example is found in the Norwegian debate, where it is argued that the Norwegian healthcare system is endangered because Norway is one of the few countries that does not have proton therapy. The argument goes like this:

P1 For a long time Norway was the only country together with Iceland and Albania that did not have PET (positron emission tomography).

P2 Now we are on the verge of making the same mistake with regard to proton therapy.

C Thus we should have proton therapy (in Norway). ${ }^{12}$

This argument can be interpreted in many ways. The form of some of these arguments is presented below:

P1 Only a few countries do not have $\mathrm{T}$ (proton therapy).

P2 We do not want to be compared with the countries that do not have $\mathrm{T}$.

C We should implement $\mathrm{T}$.

This is partly an argument from adverse consequences (sometimes called appeal to fear and scare tactics). If we do not implement proton therapy, something bad will happen - that is, we will be in the class with those we would not like to be compared with.

However, the argument also hinges on the form:

P1 Most countries have $T$ (proton therapy).

P2 Most countries cannot be wrong.

C Therefore we should have $\mathrm{T}$.

This is an appeal to widespread belief (also called argumentum ad populum, bandwagon argument, peer pressure and appeal to common practice) because it refers to common practice or what everybody does or believes.

Other similar arguments can be identified as well.

A short and more general version of the argument goes like this:
P1 We do not know whether $\mathrm{T}$ is good.

P2 If we do not implement $\mathrm{T}$, something worse will happen.

C It is good to implement $T$.

This can be called avoiding worse alternative argument. The problem is that it is far from obvious that something worse will happen, which makes it a non sequitur argument.

Furthermore, this prolific argument can also be interpreted and formulated as a slippery slope argument (with a straw man fallacy):

P1 If A occurs (we do not implement proton therapy), B is more likely to occur (we are one of few countries that do not have proton therapy).

P2 B is bad.

C Therefore A is bad (ie, it is bad not to implement proton therapy).

There are many challenges with the slippery slope argument. Most prominently, it is questionable whether A will actually lead to $\mathrm{B}$, and whether $\mathrm{B}$ is as bad as claimed. ${ }^{14}$

\section{AVOIDING RCTS}

Another interesting argument promoting proton therapy by opposing randomised trials is the argument that such trials are unnecessary or controversial. "Even when RCTs are available, rarely do they provide all the information that is needed to care most effectively for the patient. We make informed evaluations of the level of confidence one has in any given judgment ..., and we must base our actions on information about which we have good confidence." ${ }^{\prime 6}$

The form of this argument is:

P1 "We know things with varying levels of confidence", 6 and RCTs are but one provider of confidence.

P2 RCTs do not provide the necessary information for deciding on proper treatment.

P3 We are quite confident that proton therapy is the proper treatment for (a certain group of) cancer patients (by other sources of confidence).

C Therefore, we should use, pursue and promote $\mathrm{T}$.

This argument can be interpreted in many ways. First, it has affinity to the fallacy of equivocation, as (epistemic) confidence can be confused with evidence. Furthermore, it is prone to the fallacy of extended analogy (as well as the argument by generalisation), as the analogy of decision-making in clinical practice is extended to technology assessment. It can also be interpreted as a fallacy of extension (straw man), as few would argue that RCTs will provide the one and only answer in the technology assessment of proton therapy or "that there is effectively no clinical knowledge except that learned from RCTs". ${ }^{6}$ Additionally, the argument can be related to moving the goalposts (also called raising the bar) by claiming that RCTs represent a utopian standard of evidence. Pointing to other forms of evidence and to profound challenges with RCTs, one tries to avoid the demand for evidence from RCTs. This is connected to diversionary tactics-for example, the argument by inflation of conflict: as there is disagreement on the issue of RCTs, and other sources of confidence are readily provided for, RCTs for proton therapy are neither sufficient nor necessary.

\section{REVERSED BURDEN OF PROOF}

Another argument is that proton therapy is so promising that the burden of the proof of its efficiency should be placed on the shoulders of the advocates of $x$-ray therapy and not on those of the proponents of proton therapy. "Once proton beam therapy has become clinically available, is not the burden of proof on 
conventional $x$-ray therapy? Should not its advocates have to demonstrate that the cost savings achieved by using $x$ rays are not accompanied by undesirable additional morbidity? Do the users of $x$-ray therapy have the evidence to support such a claim?"6

It is clear that proton therapy is more expensive than $x$-ray therapy, but this is outweighed by the expected reduction in morbidity. Therefore, the advocates of traditional therapy should demonstrate that the cost savings achieved by using $x$ rays compared with proton therapy are not accompanied by undesirable additional morbidity. ${ }^{6}$ This is a complex argument, but in part it is a petitio principii and an argument of reversed burden of proof. By transferring the burden of proof to the opponent, the proponent expects to win by default. It can also be seen as an argument by changing the subject (also called digression, red herring, misdirection and false emphasis).

\section{INCLINATION TOWARDS THE NEW}

This shows that part of the debate on proton therapy contains flawed elements and that these are general flaws particularly recognised in debates on technology. Does this mean that there are no sound arguments for proton therapy? Of course not. There may be many sound arguments for proton therapy, but they have not been part of the debate so far.

What does this say about attitudes to proton therapy? As Holm and Takala have pointed out, whereas the precautionary principle and the slippery slope argument are overly negative, the hopeful principle and the automatic escalator are exceedingly positive. Hence, the flawed arguments can result from an eagerness to implement proton therapy. This eagerness can stem from vested interests, but it can also derive from a genuine interest in helping suffering patients.

Hence, the reason the flawed arguments are so prominent in the field of technology implementation may be because technology implementation involves uncertainty, which makes it prone to optimistic or pessimistic attitudes leading to a tendency to accept unsound reasoning and flawed arguments.

This propensity may be rooted in a general fallacy at the basis of the positive arguments, which has been called the appeal to novelty (or argumentum ad novitatem). Belief in the new compared with the old seems to be deeply rooted in our culture, ${ }^{15-17}$ and so is the belief in technology, where even diseases gain prestige through technology. ${ }^{18}$

On the other hand, appeal to tradition (or proof from tradition, appeal to common practice, argumentum ad antiquitatem) seems to be at the basis of the negative arguments, and is often found in debates on technology. Sceptics are prone to this argument.

In both perspectives, the main problem is how uncertainties and moral dilemmas are handled: either they become insurmountable or they are ignored.

Hence, enthusiasts (and sceptics) may do more harm than good. By using flawed arguments, they may lose their trustworthiness and standing and give the opponents easy points. Flawed arguments for proton therapy may result in suboptimal cancer treatment. This happens if the technology is implemented on false premises or if it is rejected on the basis of an assessment of the quality of the arguments and not of the technology. Patients deserve the best technology available, on the basis of the best available evidence and also on the basis of the best arguments.

Competing interests: None declared.

Provenance and peer review: Not commissioned; externally peer reviewed.

\section{REFERENCES}

1. Holm S, Takala T. High hopes and automatic escalators: a critique of some new arguments in bioethics. J Med Ethics 2007;33:1-4.

2. Olsen DR, Bruland ØS, Frykholm G, et al. Proton therapy: a systematic review of clinical effectiveness. Radiother Oncol 2007;83:123-32.

3. Schulz-Ertner D, Tsujii H. Particle radiation therapy using proton and heavier ion beams. J Clin Oncol 2007;25:953-64.

4. Lodge M, Pils-Johannesma M, Stirk L, et al. A systematic literature review of the clinical and cost-effectiveness of hadron therapy in cancer. Radiother Oncol 2007:83:110-22

5. Brada M, Pils-Johannesma M, De Ruysscher D. Proton therapy in clinical practice: current clinical evidence. J Clin Oncol 2007;25:965-70.

6. Goitein M, Cox JD. Should randomized clinical trials be required for proton radiotherapy? J Clin Oncol 2008;26:175-6.

7. Lewis BE. On equipoise and emerging technologies. J Clin Oncol 2008;26:2590.

8. Macbeth FR, Williams MV. Proton therapy should be tested in randomized trials. J Clin Oncol 2008;26:2590-1.

9. Glatstein $\mathbf{E}$, Glick J, Kaiser L, et al. Should randomized clinical trials be required for proton radiotherapy? An alternative view. J Clin Oncol 2008;26:2438-9.

10. Glimelius $\mathbf{B}$, Montelius A. Do we need the randomized trials and can we do them? Radiother Oncol 2007:83:105-9.

11. Hall EJ. Intensity-modulated radiation therapy, protons, and the risk of second cancers. Int J Radiat Oncol Biol Phys 2006;65:1-7.

12. Vogt Y. Modern cancer treatment. Apollon 2008;4:22-5. (University of Oslo science magazine.)

13. Hofmann B. On value-judgments and ethics in health technology assessment. Poiesis and Praxis 2005:3:277-95.

14. Volokh E. The mechanisms in slippery slope arguments. Harv Law Rev 2003;116:1026-134.

15. Nisbet R. History of the idea of progress. New York: Basic Books, 1980.

16. von Wright GH. Myten om framsteget [The myth of progress]. Stockholm: Albert Bonniers Förlag, 1994

17. Hofmann B. The myth of technology in health care. Sci Eng Ethics 2002;8:17-29

18. Album D, Westin S. Do diseases have a prestige hierarchy? A survey among physicians and medical students. Soc Sci Med 2007;66:182-8. 